ISSN: 0258-9702 ISSN L: 2710-7647

Volumen: 30 Fecha de recibido: 25/7/2019 Fecha de publicación: Enero-Junio de 2020

Año: 2020 Fecha de aceptado: 28/8/2019 Correo: revista.sciencia@up.ac.pa

Numero: 1 Número de páginas: 1-5 URL: https://revistas.up.ac.pa/index.php/scientia

\title{
UNA REVISIÓN DE LOS ECTOPARÁSITOS DE SERPIENTES EN PANAMÁ
}

\section{LILLIAN DOMÍNGUEZ ${ }^{1}$, JOHN CLEGHORN ${ }^{2}$, SERGIO E. BERMÚDEZ C. ${ }^{1+}$}

${ }^{1}$ Instituto Conmemorativo Gorgas de Estudios de la Salud, Departamento de Investigación en Entomología Médica, Panamá, Panamá

sbermudez@gorgas.gob.pa

${ }^{2}$ Universidad de Panamá, Panamá, Panamá revista.sciencia@up.ac.pa

${ }^{1+}$ Instituto Conmemorativo Gorgas de Estudios de la Salud, Departamento de Investigación en Entomología Médica, Panamá, Panamá

\section{sbermudez@gorgas.gob.pa}

\section{RESUMEN}

En este trabajo se compila la información disponible sobre ácaros ectoparásitos de serpientes en Panamá. Se enlistas seis especies de ácaros, pertenecientes a los Órdenes Trombidiformes, Mesostigmata e Ixodida.

\section{PALABRAS CLAVES: Serpientes, ácaros, ectoparásitos, Panamá}

\section{INTRODUCCIÓN}

Las serpientes están expuestas a una amplia variedad de parásitos, sean internos como externos y, a semejanza de otros vertebrados, el grado de afectación puede variar de distintas maneras. Entre los parásitos externos, los ácaros constituyen el grupo más conocido, ya que existen escasos reportes de insectos hematófagos de este grupo de reptiles. La diversidad de 
ácaros que parasitan reptiles incluye a los géneros Eutrombicula, Ophioptes, Fonsecia (Ordens Trombidiformes), Ophiogonylus, Ophionyssus, Zeterhercon (Orden Mesostigmata), Amblyomma, Bothriocroton, Haemaphysalis, Ixodes, Ornithodoros (Orden Ixodida). En serpientes el parasitismo se da en condiciones naturales, incluyendo en serpientes marinas, y de cautiverio, siendo este último el que provoca los mayores efectos nocivos en los animales.

Debido a que para Panamá existen pocos reportes de ácaros no Ixodida que parasitan serpientes, en este trabajo se compiló la información documentada en artículos científicos depositados en bases de datos de literatura científica (BioOne, Smithsonian Libraries, and PubMed), teniendo como palabras claves "ectoparásitos”, “serpientes”, “ácaros”, "garrapatas", y sus variantes en inglés "ectoparasites", "snakes", "mites", "ticks",

"Squamata", "Serpientes". Adicionalmente se revisó el material depositado en el Acervo de Ectoparásitos de la Colección Zoológica "Dr. Eustorgio Méndez" del Instituto Conmemorativo Gorgas de Estudios de la Salud (CoZEM-ICGES).

\section{ORDEN TROMBIDIFORMES}

\section{Familia Trombiculidae}

Eutrombicula alfreddugesi (Oudemans, 1910)

Esta especie conforma parte de los ácaros que popularmente se conocen como "coloradillas". Las larvas son parásitos eclécticos y se han reportado en varias especies de reptiles, aves y mamíferos. Fairchild et al. (1966), presenta un completo listado de los hospederos de $E$. alfreddugesi en Panamá.

\section{Serpientes parasitadas en Panamá:}

Colubridae: Oxybelis sp., Phrynonax poecilonotus, Spilotes pullatus.

Eutrombicula goeldii (Oudemans, 1910)

Similar a la especie anterior, E. goeldii se han reportado varios grupos de anfibios, reptiles, aves y mamíferos. Fairchild et al. (1966), presenta un completo listado de los hospederos de E. alfreddugesi en Panamá.

\section{Serpientes parasitadas en Panamá:}

Colubridae: Phrynonax poecilonotus. 


\section{ORDEN MESOSTIGMATA}

\section{Familia Macronyssidae}

Ophionyssus natricis (Gervais, 1844)

Esta especie es considerada una plaga de serpientes en cautiverio, además de poder parasitar facultativamente otros animales en los recintos (Rodríguez y Lazcano, 1992). Fue inicialmente descrita en serpientes de Europa y en la actualidad se ha reportado en también en África, Oceanía, Asia y en América, específicamente en México (Rodríguez y Lazcano, 1992), Nicaragua (Rimbaud et al. 2006), y Panamá (Miranda et al. 2017).

\section{Serpientes parasitadas en Panamá:}

Boidae: Boa imperator, Corallus caninus, Corallus ruschenbergerii, Epicrates maurus. Pythonidae: Python regius.

\section{ORDEN IXODIDA}

\section{Familia Argasidae}

\section{Ornithodoros puertoricensis Fox, 1947}

Esta especie fue descrita a partir de ejemplares que se colectaron de ratas domésticas, posteriormente su rango de hospederos incluyó a otros grupos de mamíferos. Como otros Ornithodoros, esta esta es una especie nidícola. En Panamá se ha reportado en varias especies de mamíferos domésticos y silvestres, humanos, sapo común y serpientes en cautiverio (Bermúdez et al. 2013, 2015, 2017).

\section{Serpientes parasitadas en Panamá:}

Pythonidae: Python bivittatus, Python regius

Boidae: Boa imperator, Epicrates marinus.

\section{Familia Ixodidae}

Amblyomma dissimile Koch, 1844

Esta especie es la garrapata más comúnmente encontrada en reptiles y anfibios de la región Neotropical; de hecho, inmaduros y adultos pueden parasitar un mismo individuo (Guglielmone y Nava 2010). Fairchild (1943) notó que altas infestaciones de esta especie ocasionaban la muerte de serpientes en cautiverio. Posteriormente, Fairchild et al. (1966) 
menciona infestaciones masivas en Bothrops atrox y en otras serpientes. Además de reptiles, A. dissimile se ha encontrado en aves y mamíferos (Guglielmone y Nava 2010).

\section{Serpientes parasitadas en Panamá:}

Colubridae: Chironius carinatus, Clelia clelia, Oxybelis sp., Phrynonax poecilonotus, Spilotes pullatus, Thalerophis richardi.

Boidae: Boa imperator, Corallus rushenbergerii, Epicrates sp.

Viperidae: Bothrops asper, Lachesis sp.

Amblyomma rotundatum (Koch, 1844)

La ecología de $A$. rotundatum es similar a la de la especie anterior, además de compartir características morfológicas (Guglielmone y Nava 2010). Los primeros reportes sobre anfibios en Panamá realizados por Jones (1972) y Andoh et al. (2015) pueden considerarse dudosos, ya que ofrecen pocos detalles sobre sus hallazgos. Recientemente Ogrzewalska y Bermúdez (2019) describen el hallazgo en una comunidad de Chepo.

\section{Serpientes parasitadas en Panamá:}

Viperidae: Bothrops asper.

La presencia de ácaros en serpientes de Panamá amerita mayores observaciones de campo, lo que permitiría comprender más el grado de afectación que les pudiera ocasionar en condiciones naturales. Por otro lado, los animales en cautiverio están propensos a ser parasitados por otras especies como $O$. natricis u $O$. puertoricensis, especies que podrían afectar también a humanos.

\section{Referencias}

Bermúdez, SE; Miranda, R; Kadoch, N. 2013. Reporte de larvas de Ornithodoros puertoricensis Fox 1947 (Ixodida: Argasidae) parasitando a Rhinella marina (L. 1758) (Anura: Bufonidae) en David, Chiriquí, Panamá. Puente Biológico (5): 81-85. 
Bermúdez, S., Miranda, R., Cleghorn, J., Venzal, J. 2015. Ornithodoros (Alectorobius) puertoricensis (Ixodida: Argasidae) parasitizing exotic reptiles pets in Panama. Revista FAVE-Ciencias Veterinarias 14: 1-5.

Bermúdez S, Castillo E, Pohlenz T, Kneubehl A, Krishnavajhala A, Domínguez L, Suárez J, López J. 2017. New records of Ornithodoros puertoricensis Fox 1947 (Ixodida: Argasidae) parasitizing humans in rural and urban dwellings, Panama. Tick and Ticks-Borne Diseases 8: 466-469. http://dx.doi.org/10.1016/j.ttbdis.2017.02.004

Fairchild G. 1943. An annotated list of the bloodsucking insects, ticks and mites known from Panama. American Journal of Tropical Medicine 23(6): 569-591.

Fairchild G, G. Kohls \& V. Tipton. 1966. The ticks of Panama (Acarina: Ixodoidea). Ectoparasites of Panama. Wenzel, R., Tipton V. (edits). Field Museum of Natural History, Chicago. Illinois. 167-207.

Guglielmone A, Nava S. 2010. Hosts of Amblyomma dissimile Koch, 1844 and Amblyomma rotundatum Koch, 1844 (Acari: Ixodidae). Zootoxa. 2541: 27-49.

Miranda R, Cleghorn J, Bermúdez S, Perotti A. 2017. Ocurrence of mite Ophionyssus natricis (Acari: Macronyssidae) on captive snakes from Panama. Acarologia 57(2): 365-368.

Orgzewalska M, Bermúdez S. 2019. Detección molecular de Rickettsia bellii en Amblyomma rotundatum Koch 1884 (Ixodida: Ixodidae) en Panamá. Tecnociencia 21(1): 41-56.

Rimbaud E., Pineda N., Luna L., Zepeda N., Rivera G. 2006. Primer reporte de Ophionyssus natricis (Arthropoda, Acarina, Macronyssidae, Gervais 1953) parasitando Boa constrictor constrictor en Nicaragua. Bol. Parasitol. Esc. Med. Vet. Univ. Nac. Costa Rica, 7, 1.

Rodríguez M.L., Lazcano D. 1992. First report of the mite Ophionyssus natricis (Acarina: Macronyssidae) from Mexico. Southwestern Nat., 37, 426. 